\title{
ANÁLISIS DE LA RENTABILIDAD EN LA PRODUCCIÓN DE PAPA BLANCA COMERCIAL EN LAS REGIONES DE HUÁNUCO Y LIMA
}

\author{
ANALYSIS OF THE PROFITABILITY IN THE PRODUCTION OF COMMERCIAL WHITE \\ POTATO IN HUÁNUCO AND LIMA REGIONS
}

\author{
${ }^{1}$ Carlos Minaya
}

\begin{abstract}
Resumen
El objetivo de la presente investigación es evaluar los niveles de rentabilidad de los productores de papa blanca comercial en las regiones de Huánuco y Lima, considerando un entorno de riesgo, empleando el método de simulación estocástica de Monte Carlo, mediante el software @risk; el cual permite analizar el riesgo e influencia de las variables explicativas y su impacto en la variabilidad o riesgo de la variable explicada - representada en este caso por el indicador: Margen bruto por hectárea -, bajo el supuesto de que las variables independientes son variables aleatorias y siguen una distribución de probabilidad específica. La hipótesis central del estudio es que la región Lima tiene más probabilidades de obtener altos niveles de rentabilidad y un mayor número de escenarios positivos que la región Huánuco en la producción de papa blanca. La metodología considera los precios en chacra, los rendimientos obtenidos por hectárea y los costos de producción por hectárea, clasificados en costos sujetos a riesgo (los costos de fertilizantes, costos de pesticidas, etcétera) y los costos determinísticos (no asociados a niveles significativos de variabilidad, y que son costos generalmente constantes en las campañas de producción). Se concluye que la región Lima presenta mayor cantidad de escenarios positivos en cuanto a niveles de rentabilidad, así como niveles mayores de rentabilidad promedio, asociado a menores niveles de riesgo. Las principales fuentes de riesgo de la producción de papa blanca, en ambas regiones, son el precio en chacra y los rendimientos por hectárea. El riesgo en la rentabilidad producto de variaciones en los precios es similar en ambas regiones, pero en el caso de los rendimientos existe una diferencia importante entre estas dos regiones, lo cual lleva a que los agricultores de Huánuco obtengan mayores probabilidades de obtener resultados económicos negativos.
\end{abstract}

Palabras clave: Papa blanca, rentabilidad, variable aleatoria, simulación estocástica, riesgo.

\begin{abstract}
The objective of this research is to evaluate the profitability of commercial white potato producers in the regions of Huánuco and Lima, considering a risk environment, using the method of stochastic Monte Carlo simulation, using the (a) risk software, the which analyzes the risk and impact of the explanatory variables and their impact on the variability or risk of the dependent variable - in this case represented by the indicator: Gross margin per hectare - under the assumption that the independent variables are random variables and are specific probability distribution .

The central hypothesis of the study is that the Lima region is more likely to achieve high levels of profitability and more positive scenarios that the Huánuco region in the production of white potato. The methodology considers farm-gate prices , yields per hectare and production costs per hectare , ranked costs subject to risk (the cost of fertilizer, pesticide costs , etc.) and the deterministic cost (not associated with significant levels of variability, and they are usually constant costs in production runs). We conclude that the region Lima presents more positive scenarios for profitability as well as higher average returns associated with lower levels of risk. The main sources of risk for white potato production in both regions are the farm price and yields per hectare. The risk on profitability product price changes is similar in both regions, but in the case of income there is an important difference between these two regions, which leads to Huánuco farmers get a better chance of negative economic results.
\end{abstract}

Key words: White Potato, profitability, random variable, stochastic simulation, risk.

\section{Introducción}

Actualmente en el Perú, la papa (Solanum tuberosum) es el principal cultivo del país en cuanto a superficie cosechada $(296,440$ hectáreas en el 2011) y representa el $25 \%$ del PBI agropecuario. Es la base de la alimentación de la zona andina y es producida por 600 mil pequeñas unidades agrarias. (MINAG, 2013). Según el MINAG (2013), el Perú es el país con la mayor diversidad de papas en el mundo, al contar con 8 especies nativas domesticadas y 2,301 de las más de 4,000 variedades que existen en Latinoamérica. 
Cabe señalar que nuestro país posee 91 de las 200 especies que crecen en forma silvestre en casi todo nuestro continente (mayormente no comestibles). En cuanto a sus características nutricionales, la papa contiene, en 100 gramos, 78 gr. de humedad, 18.5 gr. de almidón y es rico en Potasio (560 mg) y vitamina C (20 mg). En cuanto a su consumo, la papa es considerado un alimento básico para la población peruana pues el consumo per cápita de este producto alcanza los $90 \mathrm{Kg}$ por año. La papa compite con el trigo y el arroz en la dieta alimentaria del consumidor peruano (Diez, R., Gómez, R., Navarro, O., Varona, A. y Anderson, M., 2013).

Conociendo la importancia del cultivo de la papa para el país, en cuanto a su consumo y producción, el objetivo de la presente investigación es evaluar y analizar, bajo un entorno probabilístico, las ventajas de los agricultores en cuanto a los niveles de rentabilidad derivados de esta actividad productiva en las regiones de Huánuco y Lima. La primera porque en los últimos años se ha posicionado como el primer abastecedor de papa comercial en nuestro país con una producción en el año 2011 de 516,514 toneladas (Dirección Regional Agraria de Huánuco) y Lima por su dinamismo en productividad, ya que es la región donde se encuentra el mayor rendimiento por hectárea a nivel nacional (23.9 t/ha en el año 2011 versus $13.7 \mathrm{t} /$ ha a nivel nacional) (Portal agrario del MINAG); es decir, obtiene casi el doble de rendimiento por hectárea que el promedio nacional. Como objetivos específicos se determinarán los efectos de las principales variables que influyen en los niveles de rentabilidad, así como identificar a las principales fuentes de riesgo en esta actividad.

\section{HIPÓTESIS}

La hipótesis general del presente estudio sugiere que los niveles de rentabilidad esperados en la región Lima son significativamente mayores en comparación con la región Huánuco, además, se puede esperar la presencia de un mayor número de escenarios negativos para los productores de Huánuco que para los de Lima, es decir, hay un mayor riesgo de fracaso económico en dicha región para la producción de papa blanca comercial. También, se infiere que, los precios en chacra cobrados por los productores y los rendimientos por hectárea son las principales variables que originan que la producción de papa blanca se considere una actividad riesgosa para ambos grupos de agricultores.

\section{Materiales y métodos}

Simulación: Es el proceso de construir un modelo lógico-matemático de un sistema o proceso de decisión, y experimentar con el modelo para comprender el comportamiento del sistema o ayudar en la toma de decisiones y es particularmente útil en problemas o situaciones que involucran incertidumbre. Un modelo es inservible si no ayuda al usuario a comprender el problema. Por ello, el punto principal en la simulación está puesto en conducir experimentos con el modelo y analizar los resultados. (Palisade Corporation, 2013).

\section{La simulación de Monte Carlo}

Es una técnica cuantitativa que hace uso de la estadística, mediante modelos matemáticos, simulando el comportamiento aleatorio de sistemas reales no dinámicos. La clave de la simulación de Monte Carlo consiste en crear un muodelo matemático del sistema, proceso o actividad que se quiere analizar, identificando aquellas variables (inputs del modelo) cuyo comportamiento aleatorio determina el comportamiento global del sistema. Una vez identificados dichos inputs o variables aleatorias, se lleva a cabo un experimento consistente en generar muestras aleatorias (valores concretos) para dichos inputs y analizar el comportamiento del sistema ante los valores generados (PRAM Guide, 1997). Tras repetir " $n$ " veces este experimento, dispondremos de "n" observaciones sobre el comportamiento del sistema, lo cual nos será de utilidad para entender el funcionamiento del mismo, obviamente nuestro análisis será tanto más preciso cuanto mayor sea el número " $n$ " de experimentos que llevemos a cabo.

Para el caso de nuestro experimento se realiza la corrida en@risk con 10000 iteraciones, es decir, una gama alta de posibles escenarios, generando una serie de gráficos, con énfasis en las probabilidades de ocurrencia del output, que sirven para el análisis comparativo.

\section{De la aplicación del @risk:}

De acuerdo a la literatura, se sabe de la importancia de la simulación como una herramienta para el manejo de la incertidumbre. En nuestro caso, se busca entender, analizar y explicar comparativamente los resultados obtenidos en estas dos regiones de nuestro país. Para tal fin, usaremos el @risk. Existen dos aspectos claves para el éxito de las simulaciones de riesgo, en primer lugar, la identificación de las distribuciones de probabilidad adecuadas para cada una de las variables aleatorias del modelo, en esta investigación se usaron las distribuciones de tipo uniforme, normal y triangular, mientras que en segundo lugar, se debe definir adecuadamente las interdependencias del comportamiento esperado de dichas variables. En cuanto a las distribuciones de probabilidad, se sabe de la existencia de dos tipos de distribuciones de probabilidad, las continuas y las discretas; La cuales están definidas por uno o más parámetros. A continuación se detallan tres parámetros básicos:

- De forma, controla la forma básica de la distribución.

- De escala, controla la unidad de medida dentro del rango de la distribución.

- De ubicación, especifica la posición de la distribución relativa a cero en el eje horizontal. 


\section{Etapas en la simulación de Monte Carlo en @risk:}

1. Selección e identificación de las distribuciones de probabilidad de las variables en estudio. Dada la importancia de esta primera etapa en la identificación de las distribuciones de probabilidad de las variables críticas en el modelo, es necesario tener una buena base de datos (en este caso, data en series de tiempo) para cada variable, pues a partir de esto se podrá medir la importancia de los efectos que tienen en el riesgo y la rentabilidad de esta actividad productiva para cada región en análisis. La determinación de la distribución de probabilidad para las variables respectivas, en las dos regiones, se realizó a partir de las series de datos tomados de las fuentes de información secundaria y terciaria -se trabaja con un horizonte de 20 años-, ajustadas a las distribuciones de probabilidad correspondientes aplicando el @ risk.

\section{Selección aleatoria de un valor para cada variable} en estudio, asociado a su probabilidad de ocurrencia. Para esta etapa de la investigación, es necesario trabajar con software especialmente diseñados para la realización de las simulaciones estocásticas. En este trabajo de investigación se usó un software específico para trabajar el tema de análisis de riesgo: El @risk, con capacidad de generación de números aleatorios para distribuciones de probabilidad continuas y discretas.

3. Determinación del valor del indicador de rentabilidad. El indicador de la rentabilidad, bajo un enfoque de riesgos, en la producción de papa blanca comercial- va ser representada en esta investigación por el margen bruto por hectárea: $\mathrm{Mb}$-, en ambas regiones, será dada por la siguiente fórmula:

$\mathrm{Mb}=(\mathrm{P})(\mathrm{Q})-\left(\sum_{\mathrm{i}=1}^{\mathrm{n}} \mathrm{Ci}+\mathrm{Cf}+\mathrm{Cs}+\mathrm{Cp}+\mathrm{Mo}+\mathrm{Maq}\right)$

Dónde:

$\mathrm{Mb}=\mathrm{f}(\mathrm{Mb}) \rightarrow$ Valor esperado del margen bruto por hectárea(soles/ha.)

$\mathrm{P}=\mathrm{f}(\mathrm{P}) \rightarrow$ Valor esperado del precio en chacra del producto (soles/ tonelada)

$\mathrm{Q}=\mathrm{f}(\mathrm{Q}) \rightarrow$ Valor esperado de la productividad por hectárea (toneladas/Ha.)

$\mathrm{Ci}=$ Costos determinísticos (soles/Ha.)

$\mathrm{Cf}=\mathrm{f}(\mathrm{Cf}) \rightarrow$ Valor esperado de los costos de fertilizantes (soles/Ha.)

$\mathrm{CS}=\mathrm{f}(\mathrm{Cs}) \rightarrow$ Valor esperado de los costos de semillas (soles/Ha.)

$\mathrm{Cp}=\mathrm{f}(\mathrm{Cp}) \rightarrow$ Valor esperado de los costos de pesticidas (soles/Ha.)

Mo $=\mathrm{f}(\mathrm{Mo}) \rightarrow$ Valor esperado de los costos de mano de obra (soles/Ha.)

Maq $=\mathrm{f}($ Maq $) \rightarrow$ Valor esperado de los costos de la maquinaria agrícola (soles/Ha.)

$\mathrm{n}=$ número de artículos con costos determinísticos.
De esta forma, se consideran como costos determinísticos a los valores referidos a los costos los demás insumos de producción como el agua, estiércol, sacos, y otros.

4. Repetición de las etapas 2 y 3 para la determinación de la distribución de probabilidad del indicador de rentabilidad.El@risk permite la ejecución de hasta 10,000 iteraciones o selección aleatoria de los valores de las variables simuladas y sus respectivas probabilidades de ocurrencia a partir de las distribuciones de frecuencias. Si uno desea un nivel mayor de rigor se puede incrementar las simulaciones, las cuales pueden ser desde 1 hasta 100 . En este caso, en las pruebas preliminares se encontró que los resultados eran semejantes en las diferentes simulaciones por eso se trabajó con 1 sola simulación con 10,000 iteraciones. Para evaluar la rentabilidad y el riesgo en la producción de papa blanca comercial, en las dos regiones, se determinarán las siguientes medidas, para analizar los resultados: medidas estadísticas de la variable dependiente o explicada, percentiles de riesgos y el análisis de sensibilidad respectivo. Los percentiles de riesgo mostrarán la probabilidad de obtener niveles más bajos de rentabilidad neta correspondientes a cada uno de los 20 niveles de probabilidad, de 0 a $100 \%$, divididos en tramos de $5 \%$.

\section{Resultados y discusión}

Costos de producción comparados en el cultivo de papa blanca comercial

En el tabla 1 se presenta la estructura de costos de producción para ambas regiones al año 2011.

Tabla 1. Costos de producción total para las dos regiones al año 2011.

\begin{tabular}{|c|c|c|c|c|}
\hline Ítems & Huánuco & Lima & Huánuco & Lima \\
\hline $\begin{array}{ll}\text { Costos } & \mathrm{de} \\
\text { fertilizantes } & \end{array}$ & 1790.2 & 2544.4 & $17 \%$ & $21 \%$ \\
\hline $\begin{array}{l}\text { Costos } \\
\text { semillas }\end{array}$ & 900 & 3000 & $9 \%$ & $25 \%$ \\
\hline Pesticidas & 846.00 & 1386.5 & $8 \%$ & $12 \%$ \\
\hline Mano de obra & 3400.00 & 2373.5 & $33 \%$ & $20 \%$ \\
\hline Maquinaria & 560.00 & 1237.5 & $5 \%$ & $10 \%$ \\
\hline otros costos & 2831.95 & $1,455.20$ & $27 \%$ & $12 \%$ \\
\hline $\begin{array}{l}\text { Costo total de } \\
\text { producción }\end{array}$ & 10328.15 & 11997.097 & $100 \%$ & $100 \%$ \\
\hline
\end{tabular}

A partir de esta información se observa que los costos más importantes en la producción de papa blanca comercial en Huánuco son la mano de obra y los costos determinísticos u otros costos - se incluyen en esta categoría a los costos de los otros insumos como el agua, estiércol, los gastos indirectos, gastos de imprevistos, etcétera.

En el caso de la región Lima, los mayores costos de producción se deben a las semillas y a los fertilizantes. Resalta el hecho de que en la región Lima los costos de maquinaria sean el doble que en la región de Huánuco. 
Es importante mencionar que en ambas regiones el nivel tecnológico empleado es el de tecnología media y que en ambos casos se considera al agricultor como propietario de la tierra, es así que los costos de alquiler de terreno agrícola no son tomados en cuenta para ambas regiones.

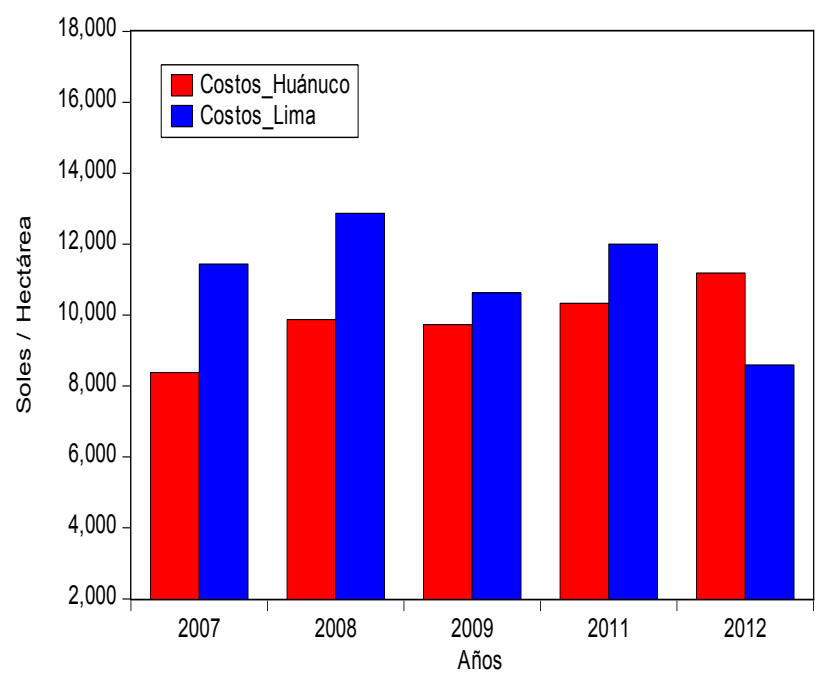

Figura 1. Costos totales de producción por hectárea en ambas regiones.

Fuente: Elaboración propia

En la fig. 1 se puede apreciar la evolución de los costos de producción por hectárea en ambas regiones. Para el caso de Huánuco los costos son el promedio regional, considerándose todas las provincias productoras de este cultivo.

Se obtuvo la información de la Dirección regional agraria de Huánuco, mientras que para el caso de Lima, se obtuvo información de las direcciones de información agraria de las provincias de Cañete, Huaral y Barranca. Se observa que los costos de producción son mayores en Lima para casi todos los años. Sólo son menores en el año 2012.

\section{Parámetros de las distribuciones de probabilidad de las variables}

Como se puede ver en el tabla 2, estos parámetros son distintos de acuerdo a la distribución de probabilidad específica de cada variable del modelo, es decir, cada conjunto de parámetros representa el comportamiento estocástico de cada una de las variables en el modelo.

Estos parámetros se obtuvieron con el software@ @risk teniendo como base la naturaleza de los datos obtenidos para así ajustar una distribución adecuada a dichas variables según los parámetros de forma, escala y de ubicación. Estos parámetros nos muestran un resumen de la distribución de cada variable, por ejemplo nos muestran el valor mínimo, el valor máximo, el valor esperado, la varianza, etcétera, que son medidas representativas de las distintas distribuciones y que determinan la probabilidad de ocurrencia del output en distintos escenarios.

Tabla 2. Parámetros de las distribuciones de probabilidad de las variables del modelo.

\begin{tabular}{ccccccc}
\hline & \multicolumn{3}{c}{ Huánuco } & \multicolumn{2}{c}{ Lima } \\
\hline Ítem & Unidades & $\begin{array}{c}\text { Distribución } \\
\text { de prob. }\end{array}$ & Parámetros & Unidades & $\begin{array}{c}\text { Distribución de } \\
\text { probabilidad }\end{array}$ & Parámetros \\
\hline Precio en chacra & $\mathrm{S} / . / \mathrm{Kg}$ & Uniforme & $0.47 ; 0.66$ & $\mathrm{~S} / \mathrm{Kg}$ & Uniforme & $0.29 ; 0.74$ \\
Rendi-miento & $\mathrm{Kg} / \mathrm{ha}$ & Uniforme & 12,$855 ; 14,615$ & $\mathrm{Kg} / \mathrm{ha}$ & Uniforme & $14172 ; 26,855$ \\
Fertilizan-tes & $\mathrm{S} / . / \mathrm{ha}$ & Uniforme & $1233 ; 2682$ & $\mathrm{~S} / \mathrm{ha}$ & Uniforme & $1989.88,4224$ \\
Semillas & $\mathrm{S} / . / \mathrm{ha}$ & Uniforme & $900 ; 1350$ & $\mathrm{~S} / \mathrm{ha}$ & Uniforme & $1600 ; 3000$ \\
Pesticidas & $\mathrm{S} / . / \mathrm{ha}$ & Triangular & $820 ; 915.5 ; 1035$ & $\mathrm{~S} / \mathrm{ha}$ & Normal & $1100.77 ; 221.54$ \\
Mano de obra & $\mathrm{S} / . / \mathrm{ha}$ & Uniforme & $1690 ; 3400$ & $\mathrm{~S} / \mathrm{ha}$ & Uniforme & $1598.84 ; 2785$ \\
Maquina-ria & $\mathrm{S} / . / \mathrm{ha}$ & Uniforme & $400 ; 560$ & $\mathrm{~S} / \mathrm{ha}$ & Uniforme & $706.65 ; 1237.5$ \\
\hline
\end{tabular}

Fuente: Elaboración propia.

\section{RESULTADOS OBTENIDOS}

En el tabla 3 se muestran las medidas estadísticas, obtenidas mediante el software@ @risk, sobre la rentabilidad en la producción de papa blanca comercial en ambas regiones. En base a 10000 iteraciones, se obtuvieron las variables de salida, los indicadores de rentabilidad, y se puede apreciar la diferencia entre ambas regiones, pues mientras Lima obtiene niveles de rentabilidad promedio ascendentes a S/./ ha 2854.94 , Huánuco sólo obtiene S/./ha 156.34. El valor mínimo del margen bruto por hectárea, lo que implica el escenario más adverso (100\% de riesgo) para la región Huánuco es de S/./ha -2224.27, mientras que en Lima se obtiene S/./ ha -4311.52 . Por otro lado, el máximo valor de margen bruto por hectárea es de $\mathrm{S} / . /$ ha 2828 para la región de Huánuco y de S./ha 12216.71 para Lima.

El riesgo de variación en el margen bruto por hectárea es más elevado en la producción de Huánuco que en Lima. Esto se verifica por el valor de la desviación estándar mayor en Huánuco.

El coeficiente de variación también es más elevado en el caso de Huánuco y es de 6.35 (y de 0.12 para Lima), lo que indica que para obtener una unidad más de rentabilidad (S/.1 adicional) es necesario arriesgar 6.35 unidades de riesgo (o sea, es necesario arriesgar $\mathrm{S} / .6 .35$ soles, puesto que el coeficiente de variación es una medida relativa de dispersión y no tiene unidades de medida). Esta medida proporciona una base más significativa de comparación 
cuando los rendimientos esperados sobre dos alternativas no son los mismos.

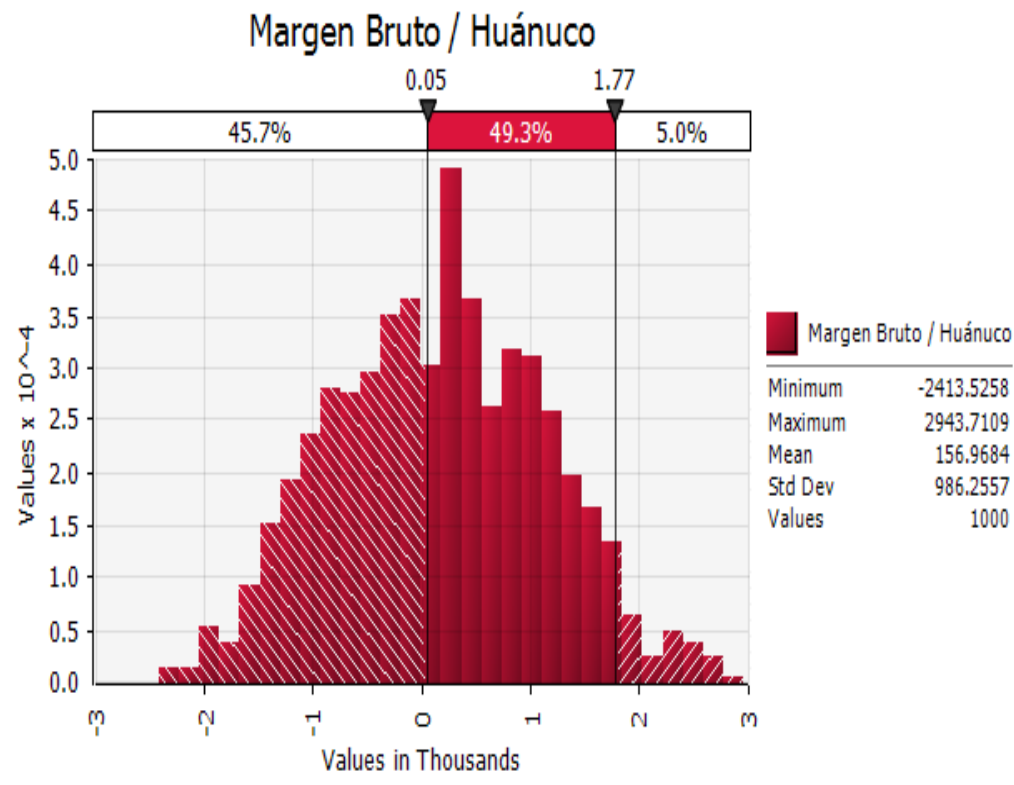

Figura 2. Distribución de probabilidad para el margen bruto por hectárea en la región Huánuco.

Fuente: Resultados de la simulación con@risk

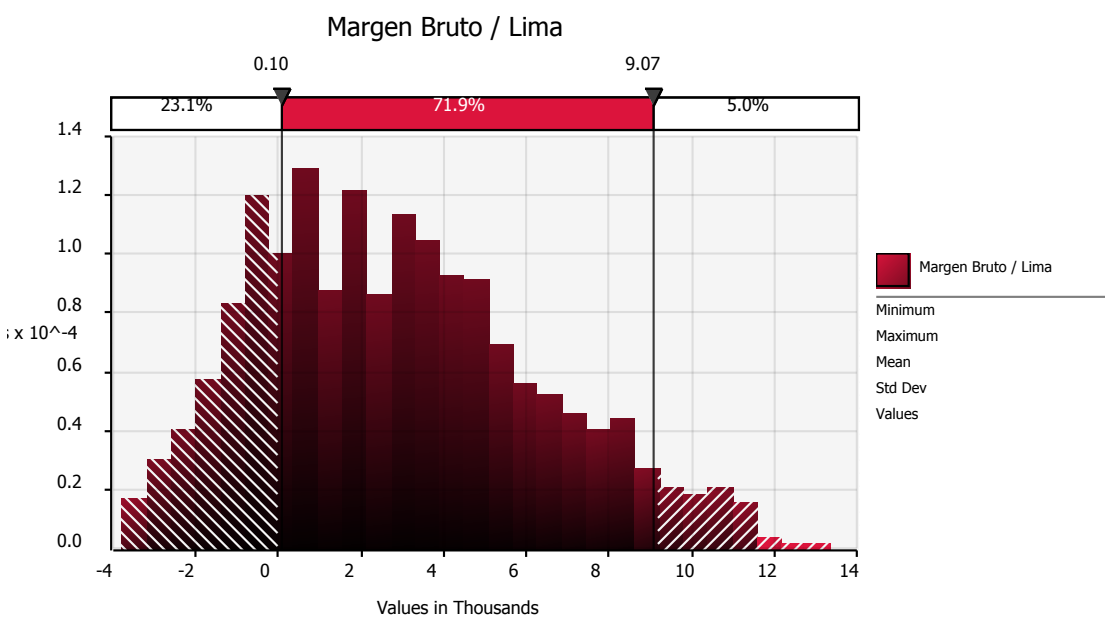

Figura 3. Distribución de probabilidad para el margen bruto por hectárea en la comparación con Huánuco, además región Lima.

Fuente: Resultados de la simulación con@risk

En laFig. 2, se observa que las probabilidades de ocurrencia en el margen bruto por hectárea de la producción de papa blanca comercial en Huánuco se encuentra distribuida entre S/./ha -2413.52 (pérdidas) y S/./ha 2934.71, con una media de 156.96 Nuevos Soles por hectárea, es decir, hay muchos escenarios de rentabilidad posibles. Al mismo tiempo, se observa que las probabilidades de ocurrencia son altas para los resultados negativos: Las probabilidades de obtener pérdidas (obtener márgenes menores a cero) son $45.7 \%$ mientras que las de obtener beneficios positivos son $54,3 \%$, pero sólo $49.3 \%$ se pueden considerar realmente posibles y un $5 \%$ de nivel de confianza es para eventos poco probables de obtener niveles de rentabilidad positivos entre S/./ha 1770 y S/./ha 2943.71.

Estos resultados nos muestran que en Huánuco es más probable que se registren pérdidas económicas para los agricultores de papa comercial, de acuerdo con los posibles valores, representados por la probabilidad de ocurrencia y distribución de probabilidad de variables importantes como los precios y rendimientos por hectárea, además de los costos de producción, como son los costos en mano de obra, maquinaria, semillas, etcétera.

En la Fig. 3, se observa que las probabilidades de ocurrencia del margen bruto por hectárea de la producción de papa blanca blanca comercial en Lima se encuentra distribuida entre $\mathrm{S} / . /$ ha $3,772.12$ y $\mathrm{S} / . /$ ha $13,359.72$, con una media de S/.2,868.32, mostrando la amplia gama de escenarios de rentabilidad posibles. Al mismo tiempo, se observa que las probabilidades de ocurrencia son altas para los resultados positivos. Las probabilidades de obtener ganancias en Lima (márgenes mayores a cero) son de 76.9\% mientras que las de obtener beneficios positivos en Huánuco son de 54.3\% (en ambos casos se suman los eventos ${ }_{13}^{-3}$ poco probables situados en el 5\% ${ }_{33}^{28}$ a la derecha). Estos resultados nos 10 muestran que en Lima también es posible que se registren pérdidas económicas para los agricultores de papa comercial, no obstante, las probabilidades de ocurrencia de pérdidas son significativamente menores $(21.3 \%<45.7 \%)$ en
comparación con Huánuco, además que es de esperar obtener beneficios económicos en esta región de acuerdo con las fluctuaciones de variables importantes como precios y rendimientos por hectárea, además de los costos de producción, como son los costos en mano de obra, maquinaria, semillas, etcétera. 
Tabla 3. Medidas estadísticas relativas al margen bruto por hectárea para ambas regiones.

\begin{tabular}{ccc}
\hline Medidas & $\begin{array}{c}\text { Región } \\
\text { Huánuco }\end{array}$ & Región Lima \\
\hline Numero de Iteraciones & 1000 & 1000 \\
Media & 156.34 & 2854.94 \\
Desviación estándar & 992.13 & 340.19 \\
Moda & -56.23 & -219.38 \\
Varianza & 984330.8 & 115738 \\
Mínimo & -2224.27 & -4311.52 \\
Máximo & 2828.91 & 12216.71 \\
Coeficiente de variabilidad & 6.35 & 0.12 \\
\hline Fuente: Elaboración propia & &
\end{tabular}

Análisis de sensibilidad: En el tabla 4 se correlacionan los resultados de las medidas de riesgo, es decir, la probabilidad de obtener niveles más bajos de rentabilidad neta correspondientes a cada uno de los 20 rangos de probabilidad de 0 a $100 \%$ divididas en clases de $5 \%$.

En el tabla 4 se puede observar que de manera general, a partir de un nivel de riesgo de $85 \%$ y descendentemente, los niveles de rentabilidad para la producción de papa en Lima son superiores que en la región Huánuco. Por ejemplo, con un nivel de riesgo de 5\% (un escenario casi libre de riesgo que representa la situación más favorable para todas las variables del modelo, incluyendo obviamente a la variable de salida), el margen bruto por hectárea máximo que se puede obtener en Lima es de S/.
8980.89, mientras que en Huánuco este mismo indicador alcanza un nivel de S/. 1811.23.

Tabla 4. Percentiles de riesgo del margen bruto por hectárea para ambas regiones.

\begin{tabular}{ccc}
\hline $\begin{array}{c}\text { Nivel de Riesgo } \\
\text { Región }\end{array}$ & $\begin{array}{c}\text { Margen bruto } \\
\text { Huánuco }\end{array}$ & $\begin{array}{c}\text { Margen bruto } \\
\text { Lima }\end{array}$ \\
\hline $100 \%$ & -2224.27 & -4311.52 \\
$95 \%$ & -1468.11 & -1943.42 \\
$90 \%$ & -1125.35 & -1270.76 \\
$85 \%$ & -893.95 & -662.96 \\
$80 \%$ & -720.95 & -239.37 \\
$75 \%$ & -539.60 & 104.63 \\
$70 \%$ & -428.72 & 479.70 \\
$65 \%$ & -293.31 & 1063.42 \\
$60 \%$ & -141.33 & 1562.04 \\
$55 \%$ & -24.16 & 1943.55 \\
$50 \%$ & 123.50 & 2436.59 \\
$45 \%$ & 271.00 & 3000.68 \\
$40 \%$ & 401.40 & 3527.20 \\
$35 \%$ & 542.42 & 3950.42 \\
$30 \%$ & 696.06 & 4538.66 \\
$25 \%$ & 849.69 & 5141.34 \\
$20 \%$ & 1024.61 & 5917.44 \\
$15 \%$ & 1238.57 & 6654.85 \\
$10 \%$ & 1493.13 & 7600.60 \\
$5 \%$ & 1811.23 & 8980.89 \\
$0 \%$ & 2828.91 & 12216.71 \\
\hline
\end{tabular}

Fuente: Elaboración propia en base a la salida del software@risk

Tabla 5: Factores de riesgo en el margen bruto por hectárea.

\begin{tabular}{lcclcc}
\hline & Región Huánuco & & \multicolumn{2}{c}{ Región Lima } \\
\hline Variables & Contribución (\%) & $\begin{array}{c}\text { Coeficiente de } \\
\text { Correlación }\end{array}$ & Variables & $\begin{array}{c}\text { Contribución } \\
(\%)\end{array}$ & $\begin{array}{c}\text { Coeficiente de } \\
\text { Correlación }\end{array}$ \\
\hline Precio en chacra & $76 \%$ & 0,788 & Precio en chacra & $79 \%$ & 0,800 \\
Rendimiento & $29 \%$ & 0,295 & Rendimiento & $55.30 \%$ & 0,537 \\
Costos de semillas & $1.30 \%$ & $-0,182$ & Costos de semillas & $1.24 \%$ & $-0,154$ \\
Costos de pesticidas & $4.50 \%$ & $-0,110$ & Costos de pesticidas & $7.30 \%$ & $-0,108$ \\
Mano de obra & $5 \%$ & $-0,490$ & Mano de obra & $1.02 \%$ & $-0,150$ \\
Maquinaria & $4.60 \%$ & $-0,003$ & Maquinaria & $4.70 \%$ & $-0,027$ \\
Otros costos & $17.50 \%$ & $-0,163$ & Otros costos & $6.60 \%$ & $-0,065$ \\
\hline
\end{tabular}

Fuente: Elaboración propia en base a la salida del software @ $@$ risk

\section{Conclusiones}

Luego de la comparación, en un entorno probabilístico, de la rentabilidad que se puede obtener a partir de la producción de papa blanca comercial en las regiones de Huánuco y Lima, se puede concluir que los niveles de rentabilidad, medidos a partir del indicador: margen bruto por hectárea, son ampliamente superiores en Lima y, en contraste, los niveles de rentabilidad que se presentan en Huánuco son mayormente negativos, es decir, en la región Huánuco, los ingresos que perciben los agricultores no cubren sus costos de producción, pues aunque en ambas regiones el precio cobrado es prácticamente similar, los rendimientos en La región Huánuco son muy bajos.

Con los resultados obtenidos, también se evidencia que la región Huánuco presenta mayores niveles de riesgo en la producción de papa blanca comercial, pues los cambios que se pueden originar en las variables independientes afectan en mayor medida la variabilidad del margen bruto por hectárea, ocasionando que esta actividad sea considerada riesgosa por los agricultores de papa blanca comercial de esta región. Más aún, se evidencia que, la probabilidad de ocurrencia en los distintos escenarios evaluados en el@risk- es de que hay más probabilidades de obtener pérdidas económicas en Huánuco que en Lima. Las principales fuentes de riesgo para ambas regiones son el precio en chacra que cobran los productores por kilo de papa blanca comercial (señales del mercado, lo cual incidirá finalmente en las decisiones de producción de los agricultores precio-aceptantes, pues ni ellos ni los demandantes pueden incidir en los precio de mercado), así como la productividad o rendimiento por hectárea, y en menor medida los costos de pesticidas. 


\section{Recomendaciones}

Se considera recomendable realizar este tipo de estudios, comparando con otros cultivos como el de la papa amarilla, el cual podría ser más rentable para el productor Huanuqueño dado que está más adaptado para sus condiciones climáticas.

Además, se debe tener en cuenta para posteriores investigaciones la inclusión de los efectos de variables ambientales en el rendimiento por hectárea en ambas regiones, en especial de los efectos del cambio climático y sus impactos económicos en los agricultores de ambas regiones.

\section{Literatura citada}

Bolívar, V. 2012. Valoración económica del impacto de la temperatura y la precipitación en la producción de papa en la cuenca del río Santa. Tesis para optar el título de Ing. Ambiental-UNALM, Lima, Perú.

Belleza, J. 2011. Análisis del rendimiento y riesgo de los cultivos agrícolas en la región Huancavelica. Trabajo monográfico presentado para optar el título de economista, mediante examen profesional-UNALM, Lima, Perú.

Castro, R. y Mokate, K. 2003. Evaluación económica y social de proyectos de inversión. Páginas 20-31. Editorial Alfa Omega, Bogotá, Colombia

Departamento Nacional de Planeación - DNP (2004), Metodología de Evaluación Ex post de programas y proyectos de inversión, Bogotá, Colombia.

Diez, R., Gómez, R., Navarro, O., Varona, A. y Anderson, M. 2013. Evaluación ex ante de alternativas transgénicas en el cultivo de papa blanca comercial. Proyecto LAC Biosafety, Subproyecto Socioeconomía: Adaptación de métodos y herramientas para la evaluación de impacto socio - económico de la introducción de OVM de maíz y papa en trópicos y centros de biodiversidad Perú, Lima, Perú.

Echevarría, N. 2011. Impacto económico del uso de semilla certificada de papa (Solanum tuberosum L.) cultivar Canchán, Distrito de Huasahuasi, Provincia de Tarma, Región Junín, Campaña Agrícola 2006 - 2007. Tesis para optar el grado de magister scientiae en la especialidad de Economía Agrícola-UNALM, Lima, Perú.

Evans, J. y Olson, D. 1998. Introduction to Simulation and Risk Analysis. Página 45. Editorial Prentice Hall, Texas, EEUU.

Fellner, A. 2004. Pequeño productor agrícola: Informe de costos y aplicación del tablero de control. XXVII Congreso Argentino de Profesores Universitarios de Costos, Buenos Aires, Argentina.

Fiorito, F. 2006. La simulación como una herramienta para el manejo de la incertidumbre. Universidad del CEMA - Máster en Finanzas 2006, Barcelona, España.
Gujarati, D. 2004. Econometría cuarta edición. Editorial Mc Graw-Hill Interamericana. Páginas 23-30, México DF, México.

Guillén, L. 2013. Análisis de la rentabilidad de una papa (Solanum tuberosum) resistente a rancha (Phytophthora infestans) en Huasahuasi, Tarma, región Junín. Tesis para optar el título de Economista-UNALM, Lima, Perú.

Gorriti, J. 2003. ¿Rentabilidad o supervivencia?: La agricultura de la costa peruana. Debate Agrario, La revista agraria, Año $03 \mathrm{~N}^{\circ}$ 35, Lima, Perú.

INEI. 2009. Estado de la población peruana, Lima, Perú. León, J. 2010. Economía aplicada. Páginas 7-28. Editorial UNAC, Lima, Perú.

Luna, H. 2013. Efectos económicos de liberar papa GM. resistente a fungosas en la localidad de Mayobamba, distrito Chinchao, provincia Huánuco, región Huánuco. Tesis para optar el título de Economista-UNALM, Lima, Perú.

Martínez, R. 2010. Gestión de programas sociales: Del diagnóstico a la evaluación de impactos. División de desarrollo social, CEPAL, Bogotá, Colombia.

Meza, W. 2000. Modelo para el análisis del comportamiento dinámico del precio de la papa blanca, Lima Metropolitana: 1985-1998. Tesis para optar el grado de magister scientiae en la especialidad de Economía Agrícola-UNALM, Lima, Perú.

Wongchuig, S. 2012. Análisis de las variables que influencian en la elección del cultivo de níspero en el distrito de Coayllo, valle del rio Omas-Asia. Tesis para optar el título de Ing. Agrícola-UNALM, Lima, Perú.

Spada, A. ,Engler P. y Seiko, M. 2011. Custos, rentabilidade e risco da produção de soja transgênica brasileira e argentina. $49^{\circ}$ Congresso da Sociedade Brasileira de Economia, Administracão e Sociologia Rural, Belo Horizonte, Brasil.

Seiko, M. et al. 2008. Retorno e risco econômico no cultivo de soja convencional e transgênica na região paulista do médio paranapanema, safra 2006/07. 46 Congresso da Sociedade Brasileira de Economia Administracão e Sociologia Rural, Rio Branco-Acre, Brasil.

Velazco, J. y Velazco, J. 2012. Empleo y Protección Social Parte 3: Características del empleo agrícola en el Perú. PUCP, Lima, Perú.

\section{Páginas de internet consultadas:}

Ministerio de Agricultura: http://www.minag.gob.pe Ministerio de Economía y Finanzas: http://www.mef. gob.pe

Palisade Corporation: http://www.palisade-lta.com/ risk/simulacion monte carlo.asp

\section{Departamento Nacional de Planeación-DNP}

(Colombia): https://www.dnp.gov.co/Portals/0/archivos/ documentos/DIFP/Bpin/Metodolo_evaluacion_Expost de_prog_proys_inv.pdf 